\title{
Influencia de la deflexión superficial en pavimentos flexibles con subrasante de baja resistencia
}

\author{
The influence of surface deflection on flexible pavements \\ with low resistance subgrade \\ Gianina S.R. Massenlli ${ }^{1 *} \quad$ Cassio E.L. de Paiva ${ }^{1}$ \\ Recibido 26 de febrero de 2018, aceptado 13 de septiembre de 2018 \\ Received: February 26, 2018 Accepted: September 13, 2018
}

\begin{abstract}
RESUMEN
Uno de los problemas más comunes que afectan el dimensionamiento de pavimentos flexibles en Brasil es la elevada deformación inicial de las capas nuevas, principalmente cuando la subrasante es débil. Este problema está asociado a la cultura brasileña en establecer, en proyecto, espesores mínimos de estas capas, una vez que ese pavimento durante su servicio no resiste al ciclo completo y, por lo tanto, los servicios de mantenimiento son precoces. El presente estudio analiza las respuestas estructurales cuando las capas del pavimento hipotético y la subrasante pierden integridad. Para eso, fueron realizados análisis de sensibilidad para examinar los efectos de cómo el cambio de los parámetros de rigidez (módulo y espesor) interfieren o influyen en el comportamiento estructural del pavimento y, por consiguiente, en la vida útil al compararlos con su estado íntegro. Los resultados mostraron que los pavimentos flexibles, con subrasante débiles y dimensionados con espesores mínimos, son considerados frágiles, o sea, con bajas resistencias. Un adecuado refuerzo de la subrasante altera todo el estado tensional del pavimento, es decir: condiciona intervenciones superficiales en las capas superiores, evitando reparaciones profundas y reconstrucción. El estudio evidencia que la deformación vertical sobre la subrasante debe ser controlada en la concepción del proyecto, próxima al límite establecido en pavimentos de larga duración. De este modo reduce la deflexión en la superficie, y, consecuentemente, evita servicios de mantenimientos prematuros y no previstos, obteniendose soluciones volcada a la realidad brasileña.
\end{abstract}

Palabras clave: Deformación vertical, subrasante débil, refuerzo de la subrasante.

\begin{abstract}
One of the most common problems that affect the flexible pavement design in Brazil is their high initial deformation of the new layers, especially for weak subgrades. This problem is associated with Brazilian culture in establishing, in design, minimum thicknesses of these layers, once that pavement during its service does not resist to the complete life-cycle and, therefore, premature maintenance and rehabilitation activities. The present study analyzes the structural responses when the layers of the hypothetical pavement and the subgrade lose integrity. For that, sensitivity analyses were carried out to examine the effects of how the change of the stiffness parameters (modulus and thickness) interfere or influence the structural behavior of the pavement and, therefore, in the useful life when compared with an intact pavement. The results showed that flexible pavements, with weak subgrade and dimensioned with minimum thicknesses,
\end{abstract}

1 Universidade Estadual de Campinas (UNICAMP), FEC, Campinas, SP, Brasil.

E-mail: gsrmasse@gmail.com; celpaiva@fec.unicamp.br

* Autor de correspondencia: E-mail: gsrmasse@ gmail.com 
are considered fragile, that is, with low resistances. An adequate reinforcement of the subgrade modifies the entire tensional state of the pavement, i.e., it affects superficial interventions in the upper layers, avoiding deep repairs and reconstruction. The study shows that the vertical deformation on the subgrade must be controlled in the conception of the design, close to the limit established in long-life pavements. In this way, it reduces the deflection on the surface, and, consequently, avoids services of premature and unforeseen maintenance, obtaining compatible solutions with the Brazilian reality.

Keywords: Vertical strain, weak subgraded, subgrade reinforcement.

\section{INTRODUCCIÓN}

En los últimos años la sociedad brasileña y el sector del transporte han enfrentado una crisis económica y política sin precedentes en el país. En esta situación crítica, se vuelve necesario, viabilizar estudios técnicos que visen identificar las deficiencias existentes y proponer soluciones con mayor retorno económico, con el objetivo de mejorar la infraestructura vial, tanto en términos de mantenimiento de las carreteras ya existentes, así como también sus futuras ampliaciones, garantizando así la calidad técnica y contribuyendo al desarrollo económico en el país.

Brasil posee el modal de transporte por carretera como la principal alternativa para el transporte de cargas. Sin embargo, las autopistas pavimentadas corresponden al $12 \%$ de toda la red de vías del país y parte de esas carreteras no se consideran adecuadas para el tráfico de personas y de bienes. Este escenario es agravado por la excesiva demanda en las carreteras, aliada a la ausencia de inversiones en mantenimiento y conservación de las mismas, servicios necesarios para garantizar la seguridad y fluidez vial, y, como consecuencia, la desvalorización de la red de carreteras brasileñas [1].

El desafío de proyectar un pavimento flexible económico y más durable cada vez es mayor, pero entra en conflicto con la cultura general actualmente empleada en el país, donde la estructura de pavimento debe ser dimensionada para que las capas tengan los espesores mínimos posibles, para soportar a las tensiones y deformaciones límites, causados por la carga de tráfico considerados estables, para un período de diseño no superior a 10 años en todo el pavimento y sus capas individuales.

En este trabajo se desarrolla un estudio de caso, que busca evaluar estructuras de pavimentos fragilizados desde su concepción, representado por un pavimento hipotético eminentemente flexible y común para la realidad brasileña. De esta forma, se simuló la pérdida de los parámetros elásticos y se evaluó el comportamiento mecánico de ese pavimento. El escenario es pasar del estado de un pavimento nuevo hasta el inicio de fatiga, representada por la ocurrencia de los defectos superficiales visibles, deflexión recuperable en la superficie por encima de lo admisible, tensiones y deformaciones crecientes, en función de la reducción de la capacidad estructural de las capas y del pavimento como un todo.

El objetivo específico de este estudio es analizar la influencia de la subrasante débil o con baja resistencia en el comportamiento de pavimentos flexibles y proponer mejoras, de modo que el pavimento sea apto para soportar un ciclo de vida completo. Para ello, se compararon las respuestas estructurales al pavimento íntegro y se pudo comprender, por medio del análisis de sensibilidad, cómo el cambio de los parámetros de rigidez incide en la vida útil del pavimento y cómo pequeños ajustes pueden mejorar el desempeño del pavimento, a fin de contribuir en el dimensionamiento, subsidiar la evaluación estructural y evitar intervenciones tempranas recurrentes.

El estudio aborda aspectos relevantes que interfieren en el comportamiento estructural del pavimento, desde la concepción del pavimento nuevo hasta el momento de la necesidad de refuerzo estructural, haciéndolo económico, duradero y, por lo tanto, sustentable. Por último, contribuye con un análisis de los límites y recomendaciones establecido en pavimentos de larga duración y se proponen posibles cambios para mejorar el desempeño de ese tipo de pavimento direccionados a la realidad brasileña.

\section{REVISIÓN BIBLIOGRÁFICA}

En la guía de diseños del MEPDG [2], un pavimento flexible convencional consiste en un revestimiento 
bituminoso, inferior a $0,15 \mathrm{~m}$, de mezcla asfáltica sobre las capas de base granular, pudiendo también tener capas de subrasante tratada o estabilizada (refuerzo de la subrasante). En la guía hay dos tipos de pavimentos flexibles, el de alta rigidez (Deep-Strength Flexible Pavements) y el compuesto por múltiples capas asfálticas (Full-Deph HMA Pavements). Los dos tipos se componen de espesas capas asfálticas (revestimiento y base), bien apoyadas, generalmente sobre una subrasante tratada, estabilizada o por una capa final de terraplenada asentada sin una fundación con tres capas de suelo compactado. El pavimento está diseñado para 20 años, por clases de vías y los servicios de mantenimiento son normalmente para recuperar las condiciones funcionales.

Los manuales de proyectos internacionales [2, 3, 4 y 5] recomiendan, en el dimensionamiento de un pavimento flexible convencional, un período de diseño de 20 o 30 años. El concepto de pavimento de larga duración (superiores a 30 años) ha sido basado en la teoría del límite de la resistencia y se supone que pequeñas deformaciones, en las capas de mezclas asfálticas, pueden resistir a innumerables solicitudes. Para ello, el control en la fundación del pavimento es riguroso, para prevenir deformación permanente de la subrasante. Las experiencias internacionales muestran un límite máximo para prevenir la deformación de compresión en la parte superior de la subrasante $\left(\varepsilon_{\mathrm{v}}\right)$, de $2,00 \times 10^{-4}$, para apoyar la estructura de pavimento [4-5]. La guía francesa recomienda el control de la deflexión vertical en la parte superior de la subrasante inferior a $200 \times 10^{-2} \mathrm{~mm}[5]$.

El Manual de DNIT (2006) [6] clasifica el pavimento flexible brasileño como aquel en que todas las capas son deformables, o sea, resilientes a la aplicación de la carga, donde esa se distribuye en parcelas aproximadamente equivalentes entre las capas. Para Medina y Motta (2015) [7], este tipo de pavimento está constituido por un revestimiento asfáltico sobre una base granular o de suelo estabilizado granulométricamente.

Para Mallick y El-Korchi (2012) [3], Balbo (2007) [8], Huang (2004) [9], la tendencia a flexión de las capas es causada por las cargas de ruedas de vehículos aplicadas sobre la superficie del pavimento $\mathrm{y}$, distribuidas entre las capas subyacentes del pavimento. Es la deformación elástica o resilientes de las capas del pavimento y de la subrasante que condiciona la fatiga de las capas superficiales más resistentes, sujetas a flexiones sucesivas, según Medina y Motta (2015) [7].

Medina y Motta (2015) [7] confirman que el defecto más frecuente, en los pavimentos flexibles en Brasil, es el agrietamiento de la capa superior de concreto asfáltico. Segundo Hveem (1955), conforme citado por [7], entendió que el agrietamiento progresivo de los revestimientos se debe a la deformación elástica (resiliente) de las capas subyacentes, principalmente de la subrasante. Sin embargo, Medina y Motta (2015) [7] complementan que ese defecto es resultado de un proceso de flexión alternada de la capa del revestimiento apoyado en capas granulares.

Otro defecto común en las autopistas brasileñas son los ahuellamientos o depresión del pavimento por las ruedas (roderas), causada por la combinación de la densificación de todas las capas y de la deformación de cizallamiento repetida, denominada deformación permanente [3], [4] y [5]. Según Mallick y El-Korchi (2012) [3] esta deformación ocurre en tres niveles, el primero cuando ocurre la deformación vertical por apenas cambio de volumen, mientras que el segundo y tercero ocurren por los esfuerzos de cizallamiento seguidos de movimiento vertical y lateral.

Estos hundimientos en el pavimento o roderas, en pavimentos flexibles, resultan de las propiedades de los materiales constituyentes del pavimento, como la visco-plasticidad de las mezclas asfálticas superiores y la plasticidad natural de las demás capas subyacentes (suelo y materiales granulares), de tal modo que en algunos modelos de fatiga esa deformación plástica en la superficie está determinada por la contribución individual de cada capa [8].

Huang (2004) [9], observó las respuestas estructurales de los pavimentos flexibles de la pista de pruebas de AASHTO y el $91 \%$ de los hundimientos ocurrieron en el propio pavimento, en donde el $32 \%$ fue en la superficie, el $14 \%$ en la base, el $45 \%$ en la sub-base y el $9 \%$ restante, en la superficie de la subrasante. En este escenario se evidenció que el material granular de la sub-base no presentaba confinamiento lateral, otros factores relevantes observados fueron el aumento de los valores de 
deflexión que aumentan con la humedad contenida en las capas del pavimento y en la superficie de la subrasante y se reducen considerablemente, con el aumento de la velocidad de los vehículos.

En Brasil, el método de DNER (DNIT, 2006) [6], es el dimensionamiento oficial para pavimentos flexibles. Esta metodología tiene origen empírico, basado en los trabajos de Turnbull, Foster \& Alhvin (1966) [10], para la USACE y basados en los datos de la pista de prueba AASHTO, a fin de determinar espesores para proteger a la subrasante de deformaciones permanentes causadas por la repetición de las cargas.

El dimensionamiento se realiza por medio de un ábaco y se determina el espesor total del pavimento, en términos del material granular, para protección de la subrasante. Para ello, es necesario que: el tráfico se convierta en el número total de solicitudes del eje estándar de $80 \mathrm{kN}$, a partir de las equivalencias de cargas USACE, denominado número $\mathrm{N}$; los valores estadísticos del CBR de la subrasante y de la capa de refuerzo de la subrasante se definen para un tramo homogéneo en términos del suelo de la subrasante y así corresponder un coeficiente de equivalencia estructural para cada material o mezcla de materiales, a fin de determinar el espesor de cada capa [8].

Para fortalecer el origen empírico del método, a algún tiempo, suplementariamente se emplean las verificaciones mecanicistas, basadas en varias ecuaciones de desempeño coherentes para la realidad brasileña. De esta forma, un pavimento pasa por dos procesos distintos de dimensionamiento. En primer lugar, la estructura es dimensionada empíricamente y, posteriormente, esa misma estructura es sometida a verificación mecanicista, donde los espesores son ajustados para atender la vida de fatiga prevista.

Otro método empírico consagrado en Brasil es el Método de la Resiliencia [11]. Se puede decir que es un procedimiento mecanístico-empírico, basado en los modelos de fatiga de mezclas asfálticas, en el comportamiento resiliente de suelos finos y materiales granulares y en el cálculo de tensiones y deformaciones considera la teoría de la elasticidad no lineal adecuado a la realidad brasileña [7].
En ambos métodos es posible desarrollar el dimensionamiento de pavimentos flexibles con una subrasante de CBR entre 2 e $10 \%$, sin ninguna restricción adicional, quedando el empleo de una capa de refuerzo adicional del suelo más cualificado, totalmente por cuenta de la buena práctica del proyectista del pavimento.

Al evaluar los pavimentos flexibles, para otro ciclo de carga de tráfico, en Brasil se emplean métodos de dimensionamiento específicos. Los métodos de refuerzo estructural (por sobrecapa asfáltica) más utilizados son los procedimientos PRO 11/79 y PRO 269/94, que figuran en el manual de restauración de pavimentos asfálticos (DNIT, 2006), [12] y se basan en la deformación de la estructura existente. Se determina el espesor del refuerzo estructural en función de una deflexión admisible, a fin de alcanzar un período de diseño sin ocurrir defectos que comprometan la estructura de pavimento. El PRO269/94 también conocido como método TECNAPAV, tiene en cuenta las propiedades resilientes de materiales del pavimento, principalmente el nivel y deformación del suelo.

En lo que se refiere al conocimiento del tema, en general, la concepción del pavimento flexible convencional brasileño es diferente en relación con otros países, principalmente en relación con el período de proyecto y a los tipos de vías. La deformación elástica o resiliencia de los pavimentos flexibles brasileños es un asunto antiguo, pero todavía ha sido un problema muy común, principalmente cuando la subrasante es débil, permitiendo la ocurrencia de defectos precoces. Y como los procedimientos vigentes de restauración consideran la resiliencia existente, en los cálculos de refuerzo estructural (sobrecapas asfálticas), esta solución se vuelve espesa y costosa. Las experiencias internacionales controlan las deformaciones en la parte superior de la subrasante, ya que responde por la mayor parte de la contribución de las deflexiones totales en un pavimento. De esta forma, cuando la estructura de pavimento no recibe la influencia de la subrasante, el pavimento se vuelve durable aumentando su vida útil. En este contexto, este estudio de caso analizará la fragilidad de un pavimento flexible con una subrasante débil, comúnmente proyectado en Brasil, y el efecto del refuerzo de la subrasante como mejora desde la concepción de la estructura de pavimento. 


\section{METODOLOGÍA Y CRITERIOS DE PROYECTO}

\section{Modelos de fatiga utilizados en la verificación mecanicista}

En Brasil, en el dimensionamiento del pavimento flexible es común el análisis de los daños para determinar el agrietamiento por fatiga y deformación permanente. Los modelos de fatiga más utilizados permiten prever un número de repeticiones de carga para prevenir la fatiga $\left(\mathrm{N}_{\mathrm{f}}\right)$, en función de la tensión o deformación. Estos modelos también definen los valores límites para la deflexión recuperable en la superficie del pavimento $\left(\mathrm{D}_{0}\right)$; deformación horizontal de tracción, en la última fibra inferior, de la carpeta asfáltica $\left(\varepsilon_{t}\right)$ y deformación vertical en la parte superior de la subrasante $\left(\varepsilon_{\mathrm{v}}\right)$. Para este estudio se utilizaron modelos constantes en los manuales de proyectos, es decir, los más utilizados para un dimensionamiento, conforme a las ecuaciones (1), (2) y (3):

- Modelo para el agrietamiento por fatiga de la carpeta asfáltica por Pinto, 1980 [13] y [14]:

$$
N_{f}=6,067-10^{-10} \times \varepsilon_{v}^{-4,762}
$$

- Modelo para la deformación permanente en la subrasante por Shell KSLA, Dorman y Metcalf [13] y [15]:

$$
N_{f}=6,067-10^{-10} \times \varepsilon_{v}^{-4,762}
$$

- Modelo para deflexión superficial, conforme PRO 269/94 [12] y [13]:

$$
\log D_{a d m}=3,148-0,188 \times \log N_{f}
$$

En donde:

$N_{f}=$ Número de repeticiones de carga para prevenir la fatiga;

$\varepsilon_{t}=$ Deformación horizontal de tracción, en la última fibra inferior, de la carpeta asfáltica;

$\varepsilon_{v}=$ Deformación vertical de compresión, en la parte superior, de la subrasante;

$D_{0}=$ Deflexión recuperable en la superficie del pavimento.

El modelo de la ecuación (1) es de origen brasileño y considera las fisuras por fatiga en la carpeta asfáltica tipo down-top, éstas se inician en la parte inferior del revestimiento y se propagan hasta alcanzar a la superficie, debido al efecto de la acción de tensiones de tracción en la fibra inferior del revestimiento $\left(\varepsilon_{t}\right)[13-14]$.

La deformación vertical de la subrasante $\left(\varepsilon_{\mathrm{v}}\right)$, representada por el modelo de la ecuación (2), está basada en el supuesto de que las capas superiores de la subrasante son proyectadas y construidas de forma adecuada para que no haya ningún hundimiento en ninguna de esas capas [3]. Según Huang (2004) [9], ese criterio de falla es válido solamente cuando la deformación permanente es causada por subrasante débil y no por las capas superpuestas.

La fatiga que se genera en la deflexión del pavimento, que es medida en la superficie del pavimento, se expresa en el modelo de la ecuación (3). Este modelo es de origen brasileño y considera la deformación del pavimento, desarrollada por Preussler y Pinto, en 1994 [12].

\section{Parámetros de diseño adoptados en el estudio de caso}

Uno de los parámetros básicos necesarios para iniciar el dimensionamiento del pavimento es el número $\mathrm{N}$ característico, o sea, el tráfico convertido en cargas equivalentes al eje estándar o eje padrón a través del número equivalente de solicitudes del eje estándar de $80 \mathrm{kN}$. Los números N's considerados en este estudio corresponden a $1,34 \times 10^{7}$ (USACE) y 4,70 x $10^{6}$ (AASHTO) para un período de proyecto de 10 años.

Otro parámetro es la caracterización de la subrasante, esta puede ser determinada por valores de CBR o Módulos de Resiliencia (E), obtenidos por medio de ensayos de laboratorio o in situ. En Brasil, los suelos tropicales, utilizados para fines de pavimentación, deben ser clasificados. La clasificación MCT [6] permite retratar las peculiaridades de los suelos finos, en cuanto al comportamiento laterítico o saprolítico. Otra clasificación es la Resiliencia [6], esta posibilita calificar los suelos finos en cuanto al comportamiento mecánico en términos de deformación elástica a, conforme se presenta en la Tabla 1.

La Tabla 1 divide los suelos finos en tres tipos (I, II y III), en donde la clase I es mejor que la clase 
Tabla 1. Clasificación de los suelos finos según su Resiliencia.

\begin{tabular}{|c|c|c|c|}
\hline \multirow{2}{*}{ CBR $(\%)$} & \multicolumn{3}{|c|}{ S (\%) } \\
\cline { 2 - 4 } & $\leq 35$ & 35 a 65 & $>65$ \\
\hline$\geq 10$ & I & II & III \\
\hline 6 a 9 & II & II & III \\
\hline 2 a 5 & III & III & III \\
\hline
\end{tabular}

Fuente: Manual de Pavimentación (DNIT, 2006) [6].

III y se observa que los suelos con valores de CBR igual o inferior al 5\% se clasifican como tipo III, independientemente de la porción de limo presente en la muestra. En estos casos, el suelo posee elevada deformación, es decir, módulos de resiliencia bajos y comportamiento no laterítico.

Algunas Instrucciones de proyecto vigentes en Brasil [13] y [17] recomiendan algunas correlaciones, en cuanto al CBR y la clasificación MCT del suelo para obtener el módulo de resiliencia (E) del suelo. Para este estudio se utilizó la ecuación (4) [13], correlación para suelos con comportamiento no laterítico.

$$
E_{s l}=18 \times C B R^{0,64}(\mathrm{MPa})
$$

En este trabajo, la definición del soporte de la subrasante, se dio por un análisis de cinco niveles de CBR, correlacionados con sus módulos de resiliencia $\left(\mathrm{E}_{\mathrm{s} 1}\right), \mathrm{E}_{\mathrm{s} 11}=20 \mathrm{MPa}, \mathrm{E}_{\mathrm{sl} 2}=30 \mathrm{MPa}$, $\mathrm{E}_{\mathrm{sl} 3}=50 \mathrm{MPa}, \mathrm{E}_{\mathrm{s} 14}=80 \mathrm{MPa}$ e $\mathrm{E}_{\mathrm{s} 15}=100 \mathrm{MPa}$. Para ello, la estructura de pavimento fue dimensionada por dos métodos brasileños (DNER y Resiliencia) y fue comparada con las respuestas obtenidas por la verificación mecanicista, que se utilizó de los modelos de fatiga de las ecuaciones (1), (2) y (3). En ese contexto, el refuerzo de la subrasante es la capa del pavimento que presentó mayor diferencia en la comparación de los tres métodos, conforme el gráfico de la Figura 1.

La deformación elástica en la superficie (deflexión) sigue siendo uno de los criterios de degradación estructural más utilizados para controlar la resistencia a la fatiga del pavimento. De esta forma, la deflexión admisible $\left(D_{a d m}\right)$ o límite puede ser obtenida por la ecuación (3) [7]. En el método de resiliencia se debe considerar la deflexión admisible para obtener el espesor de la carpeta asfáltica. Sin embargo, en la verificación mecanicista, los espesores se ajustan para atender los demás puntos analizados.

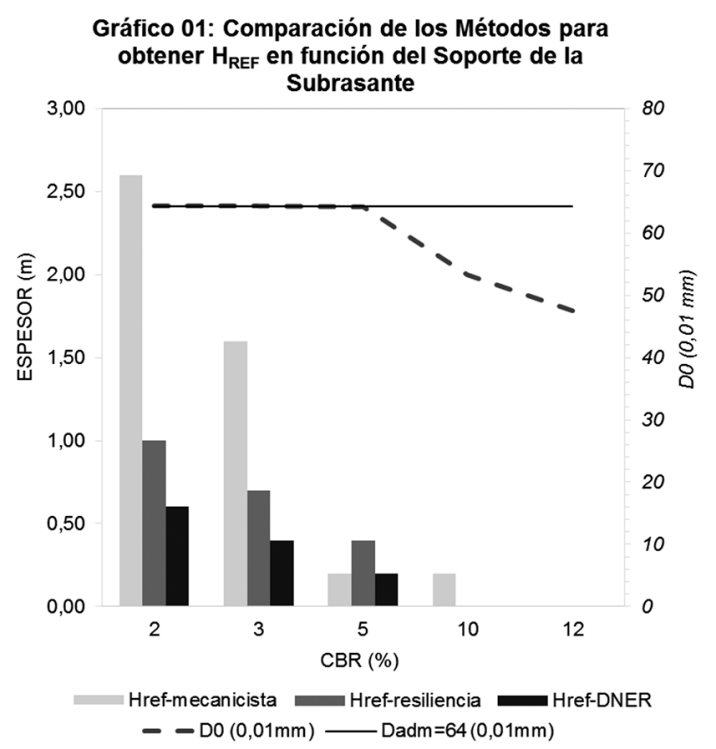

Figura 1. Determinación del espesor de refuerzo de la subrasante $\left(\mathrm{H}_{\mathrm{REF}}\right)$.

La Figura 1 está representada por dos ejes de ordenadas y, por lo tanto, dos tipos de gráficos, en función del CBR de la subrasante (eje de la abscisa). Las columnas se refieren a los espesores de refuerzo de la subrasante $\left(\mathrm{H}_{\mathrm{REF}}\right)$ representada por el eje vertical de la izquierda. Mientras que el eje de la derecha representa la deflexión $\left(\mathrm{D}_{0}\right)$, calculada en la superficie de cada suelo para una respectiva subrasante y se comprueba que:

- Para subrasante débiles con $\mathrm{CBR} \leq 5 \%\left(\mathrm{E}_{\mathrm{sl}}\right.$ $\leq 50 \mathrm{MPa}$ ) es evidente la necesidad de protección, una capa adicional de suelo más calificado, es decir, por encima de la subrasante original. Por tanto, disipar las tensiones y deformaciones con espesores de capas que ejerzan esa función;

- Los espesores $\mathrm{H}_{\mathrm{REF}}$ obtenidos directamente por el método de la Resiliencia son mayores que los del método de DNER (2006). Esta diferencia puede ser explicada porque el método de la Resiliencia considera tanto el comportamiento del suelo como también a la deformación del mismo (Tabla 1);

- Subrasantes con CBR $>12 \%\left(\mathrm{E}_{\mathrm{sl}}>100 \mathrm{MPa}\right)$ no necesitan de $\mathrm{H}_{\mathrm{REF}}$;

- En la verificación mecanicista se hizo necesario el uso de $\mathrm{H}_{\mathrm{REF}}$ para subrasante con CBR igual a $10 \%\left(\mathrm{E}_{\mathrm{sl}}=80 \mathrm{MPa}\right)$; 
- La deflexión actuante en la superficie del pavimento $\left(\mathrm{D}_{0}\right)$ evidencia una reducción acentuada en la subrasante $\mathrm{CBR}=5 \%(50 \mathrm{MPa})$.

La estructura de pavimento estudiada está constituida por revestimiento asfáltico, capas de base y sub-base granulares y refuerzo de la subrasante con suelo seleccionado (módulo de resiliencia igual a 100 $\mathrm{MPa}$ ), apoyado en un sustrato de suelo (subrasante) con módulo de resiliencia igual a $50 \mathrm{MPa}$.

\section{RESULTADOS OBTENIDOS}

\section{Estructura de referencia}

El pavimento flexible estudiado fue proyectado para 10 años, según el método empírico de DNER se utilizaron ecuaciones de previsión de fatiga, presentadas anteriormente. Se utilizó para este estudio el programa computacional de diferencias finitas, basado en la teoría de la elasticidad lineal, denominado Elsym 5, para subsidiar los cálculos y obtener las respuestas estructurales del pavimento analizado. Los datos de entrada utilizados en este programa fueron: las propiedades de las capas (espesor, módulo de resiliencia y coeficiente de Poisson), localización y magnitud de las cargas y las coordenadas de los puntos de análisis de las respuestas estructurales del pavimento. La Figura 2 presenta el modelo elástico y los espesores resultantes de la estructura de pavimento después de la verificación mecanicista.

Los módulos y coeficientes de Poisson admitidos en las capas (Figura 2) son valores medios característicos de los materiales recomendados en los guías e instrucciones de proyecto brasileños. De esta forma, los valores de los esfuerzos actuantes, obtenidos por el programa Elsym 5 fueron: $\mathrm{D}_{0}=64\left(\times 10^{-2} \mathrm{~mm}\right)$, $\varepsilon_{\mathrm{t}}=1,88\left(\mathrm{x} 10^{-4}\right)$ e $\varepsilon_{\mathrm{v}}=2,92\left(\mathrm{x} 10^{-4}\right)$. Por lo tanto, de acuerdo con los procedimientos vigentes en Brasil, la estructura de pavimento dimensionada está apta para iniciar su vida en servicio y atender un período de proyecto de 10 años.

Es importante destacar que, si la subrasante tiene un módulo de resiliencia igual a $80 \mathrm{MPa}$, el estado tensional del pavimento se alteraría, la deflexión en la superficie pasaría a $43 \times 10^{-2} \mathrm{~mm}$.

Esta deformación inicial, principalmente para una subrasante débil o frágil, puede anticipar la necesidad de servicios de restauración, ya que ese efecto interfiere en todo el pavimento. Para una mejor comprensión de este fenómeno, se realizaron análisis de sensibilidad de los parámetros de rigidez de las capas del pavimento y de la subrasante. Los análisis se dividieron en dos fases: en la primera fase se caracterizó por la pérdida de la integridad del pavimento, donde los espesores y los módulos elásticos de cada capa fueron gradualmente reducidos y la segunda fase en donde se analizó un refuerzo estructural necesario para eliminar la alta deflexión superficie del pavimento nuevo para ese mismo pavimento. Para cada estructura analizada se obtuvieron las respuestas estructurales referentes a las deformaciones horizontales de tracción en la fibra inferior del revestimiento $\left(\varepsilon_{t}\right)$, a las deformaciones verticales de compresión

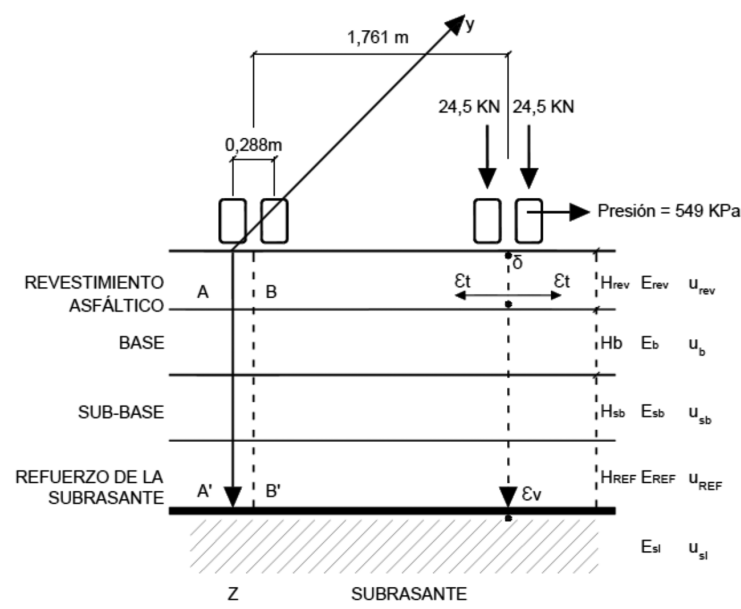

\begin{tabular}{|c|c|c|c|c|}
\hline Capa & Material & $\begin{array}{c}\text { Coef. De } \\
\text { Poisson } \\
(\boldsymbol{\mu})\end{array}$ & $\begin{array}{c}\text { Módulo } \\
\text { de } \\
\text { Elasticidad } \\
(\mathbf{M P a})\end{array}$ & $\begin{array}{c}\text { Espesor } \\
\mathbf{H}(\mathbf{m})\end{array}$ \\
\hline Revestimiento & Concreto Asfáltico & 0,30 & 3.500 & 0,13 \\
\hline Base & $\begin{array}{c}\text { Material Granular } \\
\text { (bien graduado) }\end{array}$ & 0,35 & 300 & 0,15 \\
\hline Sub-Base & Material Granul ar & 0,40 & 200 & 0,20 \\
\hline Refuerzo & Suelo seleccionado & 0,40 & 100 & 0,20 \\
\hline Subrasante & Subrasante existente & 0,45 & 50 & - \\
\hline
\end{tabular}

Figura 2. Datos de entrada y estructura del pavimento dimensionada. 
vertical en la parte superior de la subrasante $\left(\varepsilon_{\mathrm{v}}\right)$ y la deflexión en la superficie $\left(\mathrm{D}_{0}\right)$.

\section{Análisis de sensibilidad de la reducción estructural de las capas del pavimento}

Las pérdidas de integridad de las capas del pavimento se representaron por la reducción o disminución de las espesuras efectivas $(\mathrm{H})$ y de los módulos elásticos con contribución estructural (E), como se muestra en la Tabla 2.

La variación de los esfuerzos actuantes, en función de cada parámetro modificado, fue expresada en porcentaje, como fue propuesto por Mallick y ElKorchi [3]. Por lo tanto, se compararon los análisis de sensibilidad de cada esfuerzo en relación con los cambios de espesor y el módulo de resiliencia de las capas y de la subrasante.

Las Figuras 3 hasta la 6 ilustran gráficamente los tres pares de curvas resultantes para cada capa del pavimento y subrasante. El origen del sistema de coordenadas (punto 0,0 ) representa el pavimento en condiciones estructurales íntegras iniciales del pavimento nuevo con subrasante de $\mathrm{E}_{\mathrm{sl}}=50 \mathrm{MPa}$. Los valores negativos del eje de las abscisas representan la pérdida de la integridad del revestimiento. El eje de las ordenadas representan el aumento de los esfuerzos. La Tabla 3 muestra una leyenda de las designaciones.

El análisis de sensibilidad del revestimiento, presentado en la Figura 3, muestra que el parámetro espesor del revestimiento $\left(\mathrm{H}_{\mathrm{rev}}\right)$ es muy sensible a los esfuerzos de deformación $\varepsilon_{v}$ e $\varepsilon_{t}$, y moderadamente sensible a la deflexión en la superficie del pavimento $\left(\mathrm{D}_{0}\right)$.

La Figura 4 muestra la sensibilidad de la capa de base, donde $\varepsilon_{\mathrm{t}}$ es muy sensible a la variación del módulo elástico de la base $\left(\mathrm{E}_{\mathrm{b}}\right)$, así como $\varepsilon_{\mathrm{v}}$ es sensible a la variación de $\mathrm{E}_{\mathrm{b}}$. Con respecto a la sub-base, la Figura 5 ilustra que $\varepsilon_{\mathrm{v}}$ es más sensible a la variación de $\mathrm{H}_{\mathrm{sb}}$. Este escenario muestra que las pérdidas de $\mathrm{H}_{\mathrm{b}} \mathrm{y} \mathrm{H}_{\mathrm{sb}}$ contribuyen, en proporciones similares, al aumento de $\varepsilon_{\mathrm{v}}$. En las Figuras 4 y 5 , se observa que la variación de los parámetros de rigidez de la base y sub-base no es significativos para $\mathrm{D}_{0}$. Otro hecho observado, en las Figuras 3 y 4, es que los módulos $\mathrm{E}_{\mathrm{rev}}$ y $\mathrm{E}_{\mathrm{b}}$ poseen el mismo grado de importancia para $\varepsilon_{\mathrm{t}}$.

Tabla 2. Plan de pérdida de integridad.

\begin{tabular}{|l|l|c|c|c|}
\hline \multicolumn{1}{|c|}{ Capa } & \multicolumn{1}{|c|}{ Material } & $\begin{array}{c}\text { Espesor } \\
(\mathbf{m})\end{array}$ & $\begin{array}{c}\text { Módulo de } \\
\text { Elasticidad (MPa) }\end{array}$ & $\begin{array}{c}\text { Coef. de } \\
\text { Poisson }(\boldsymbol{\mu})\end{array}$ \\
\hline Revestimiento & Concreto Asfáltico & 0,13 a 0,04 & 3500 a 1100 & 0,3 \\
\hline Base & Granular bien graduada (BGS) & 0,15 a 0,10 & 300 a 100 & 0,35 \\
\hline Sub-Base & Granular (CBR $\geq 20 \%)$ & 0,20 a 0,10 & 200 a 100 & 0,4 \\
\hline Refuerzo & Suelo seleccionado $(\mathrm{CBR} \geq 10 \%)$ & 0,2 & 100 & 0,4 \\
\hline Subrasante & Subrasante existente (CBR $\geq 5 \%)$ & $\infty$ & 50 a 20 & 0,45 \\
\hline
\end{tabular}

Tabla 3. Leyenda para los gráficos.

\begin{tabular}{|l|l|}
\hline \multicolumn{1}{|c|}{ Sigla } & \multicolumn{1}{c|}{ Denominación } \\
\hline D0 & Deflexión recuperable en la superficie \\
\hline et & Deformación de tracción horizontal en la fibra inferior de las carpetas asfálticas \\
\hline ev & Deformación de compresión vertical en la parte superior de la subrasante \\
\hline $\mathrm{Hrev}$ & Espesor del revestimiento (carpeta asfáltica) \\
\hline $\mathrm{Hb}$ & Espesor de la base \\
\hline $\mathrm{Hsb}$ & Espesor de la sub-base \\
\hline Erev & Módulo del revestimiento (carpeta asfáltica) \\
\hline $\mathrm{Eb}$ & Módulo de la base \\
\hline Esb & Módulo de la sub-base \\
\hline Esl & Módulo de la subrasante \\
\hline
\end{tabular}




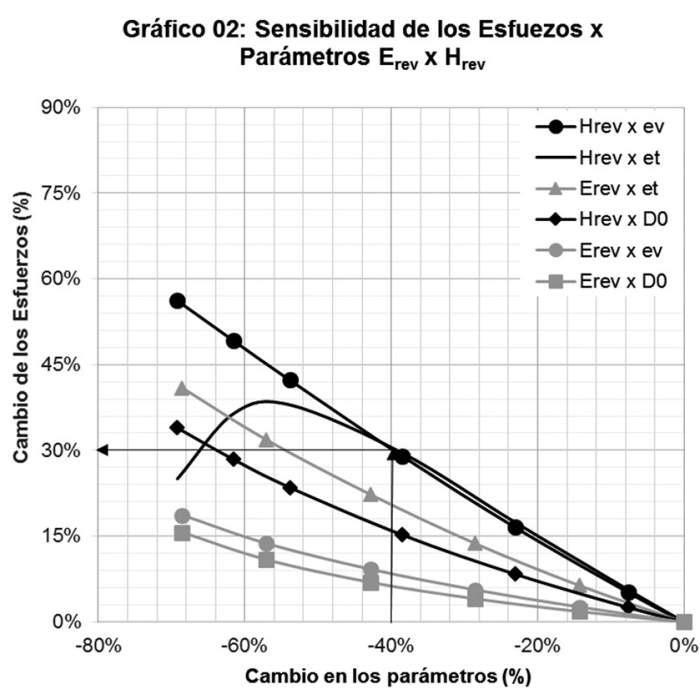

Figura 3. Sensibilidad de $\varepsilon_{\mathrm{t}}, \varepsilon_{\mathrm{v}}, \mathrm{D}_{0} \mathrm{x} \mathrm{E}_{\mathrm{rev}}, \mathrm{H}_{\mathrm{rev}}$.

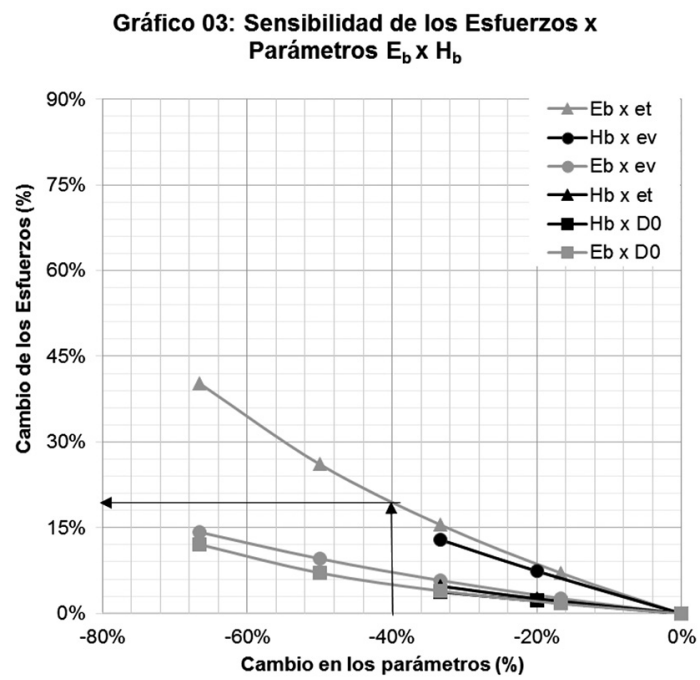

Figura 4. Sensibilidad de $\varepsilon_{\mathrm{t}}, \varepsilon_{\mathrm{v}}, \mathrm{D}_{0} \times \mathrm{E}_{\mathrm{b}}, \mathrm{H}_{\mathrm{b}}$.

El análisis de sensibilidad de la subrasante muestra que la reducción de su módulo $\left(\mathrm{E}_{\mathrm{sl}}\right)$ afecta directamente los esfuerzos $\mathrm{D}_{0}$ y $\varepsilon_{\mathrm{v}}$, como ilustran las curvas $\left(\mathrm{E}_{\mathrm{sl}} \times \mathrm{D}_{0}\right)$ y $\left(\mathrm{E}_{\mathrm{sl}} \times \varepsilon_{\mathrm{v}}\right)$ en la Figura 6. En el pavimento estudiado, la subrasante tiene una reducción de su módulo de $50 \mathrm{MPa}$ a $30 \mathrm{MPa}(-40 \%)$, su deflexión recuperable en la superficie aumenta en un $40 \%$, es decir, la deflexión superficial de 64 (x $\left.10^{-2} \mathrm{~mm}\right)$ pasa a $89\left(\mathrm{x} 10^{-2} \mathrm{~mm}\right)$. Para una mejor comprensión, se agrupó en la Figura 7 las curvas de esfuerzos en función de los parámetros de rigidez más relevantes obtenidos en los análisis anteriores.

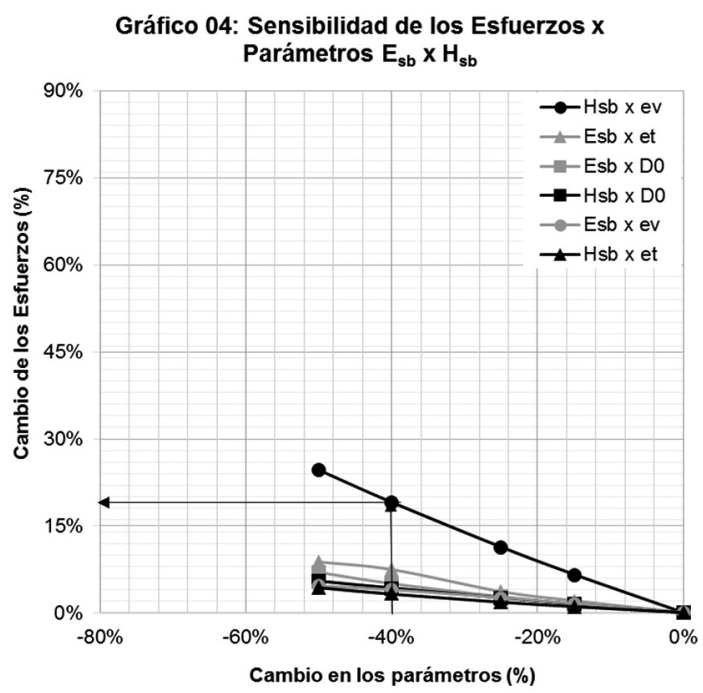

Figura 5. Sensibilidad de $\varepsilon_{\mathrm{t}}, \varepsilon_{\mathrm{v}}, \mathrm{D}_{0} \times \mathrm{E}_{\mathrm{sb}}, \mathrm{H}_{\mathrm{sb}}$.

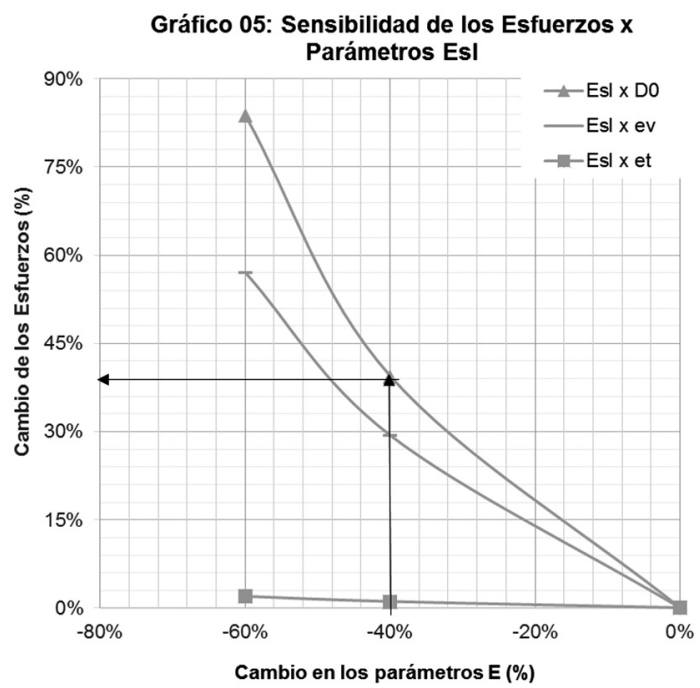

Figura 6. Sensibilidad de $\varepsilon_{\mathrm{t}}, \varepsilon_{\mathrm{v}}, \mathrm{D}_{0} \times \mathrm{E}_{\mathrm{sl}}$.

En el gráfico de la Figura 7 se puede visualizar cómo el módulo de la subrasante $\left(\mathrm{E}_{\mathrm{sl}}\right)$ se destaca en relación con los demás parámetros. Esto significa que la resistencia de la subrasante refleja directamente en la deflexión de la superficie $\left(\mathrm{D}_{0}\right)$ desde su estado nuevo y éste, a su vez, en el desempeño del pavimento y ciclos de vida. Se observa que, además de $\mathrm{D}_{0}, \varepsilon_{\mathrm{v}}$ también es directamente afectado por la reducción del módulo de la subrasante.

Se observa una gran dependencia de $\mathrm{D}_{0}$ y $\varepsilon_{\mathrm{v}}$ en relación con la variación de $\mathrm{E}_{\mathrm{sl}}$, justificando un mejor 


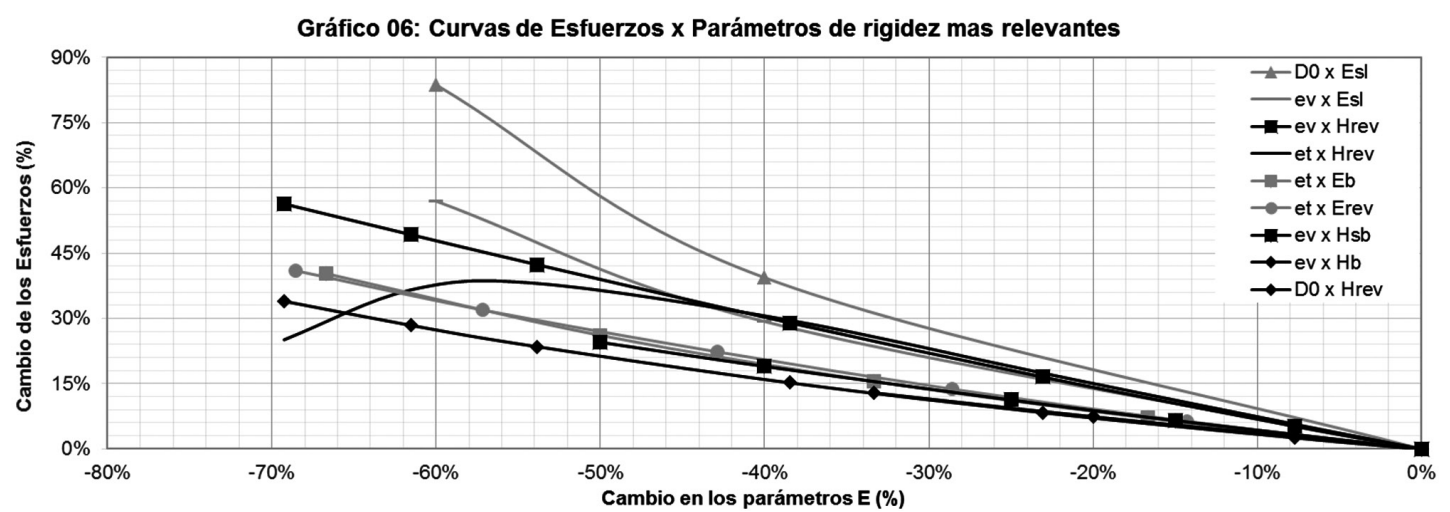

Figura 7. Curvas más relevantes de los análisis de sensibilidad.

análisis del refuerzo de esta estructura, para proteger la subrasante y, por consiguiente, inhibir la deformación de las capas superiores para mantener el pavimento con baja deflexión superficial al inicio de su vida útil.

Las curvas, representadas en la Figura 8, parten de la estructura de referencia donde $\mathrm{H}_{\mathrm{REF}}$ es igual a $0,20 \mathrm{~m}$ (punto de origen 0,0 ). Se observa que esta condición de refuerzo estructural reduce $\varepsilon_{\mathrm{v}}$ sensiblemente, seguida de $D_{0}$. Se observa que
$\mathrm{H}_{\mathrm{REF}}$ no contribuye a la reducción de $\varepsilon_{t}$, ya que esta función corresponde a las capas de base y de revestimiento. En el ejemplo estudiado, si $\mathrm{H}_{\mathrm{REF}} \mathrm{se}$ cambia de $0,20 \mathrm{~m}$ a $0,40 \mathrm{~m}$, el comportamiento del suelo mejorará considerablemente.

La Tabla 4 presenta las respuestas estructurales actuantes cuando el $\mathrm{H}_{\mathrm{REF}}$ de $0,20 \mathrm{~m}$ pasa a tener 0,40 y $0,60 \mathrm{~m}$ y cómo esos valores pueden influir en el refuerzo estructural de un pavimento.

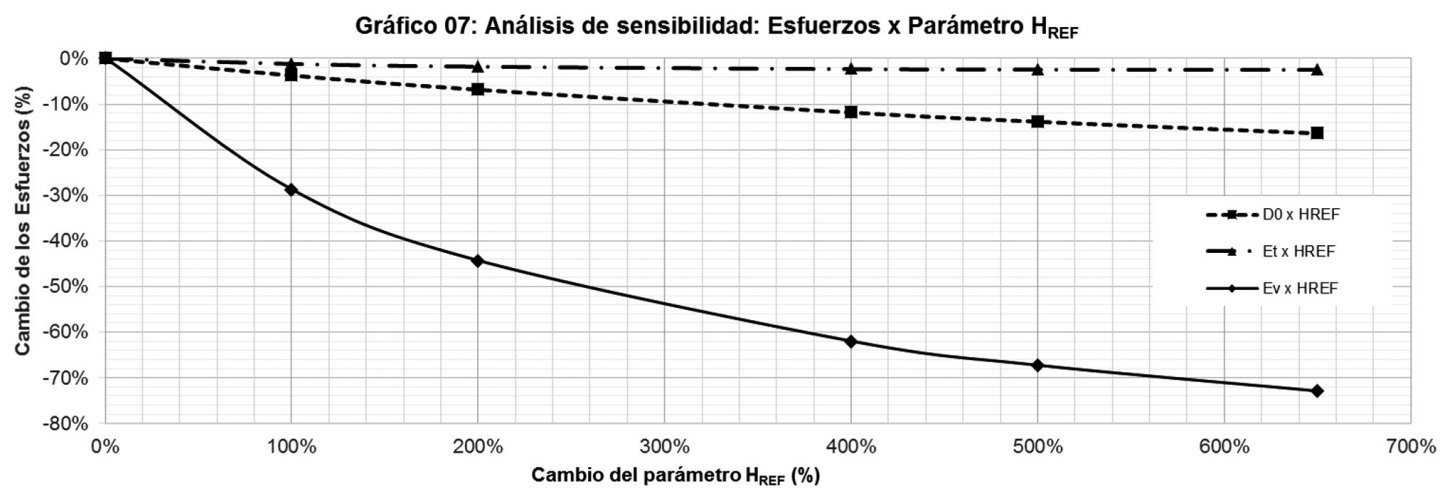

Figura 8. Sensibilidad de los esfuerzos en función de los espesores de refuerzo $\left(\mathrm{H}_{\mathrm{REF}}\right)$.

Tabla 4. Estado de tensiones del suelo de referencia $\left(\mathrm{E}_{\mathrm{sl}}=50 \mathrm{MPa}\right)$, variando $\mathrm{H}_{\mathrm{REF}}$.

\begin{tabular}{|l|c|c|c|c|}
\hline \multirow{2}{*}{ Esfuerzo } & \multicolumn{3}{|c|}{ H $_{\text {REF }}(\mathbf{m})$} & \multirow{2}{*}{ Posición de Análisis (m) } \\
\cline { 2 - 4 } & $\mathbf{0 , 2 0}(*)$ & $\mathbf{0 , 4 0}$ & $\mathbf{0 , 6 0}$ & \\
\hline $\mathrm{D}_{0}(0,01 \mathrm{~mm})$ & 64 & 62 & 60 & 0,01 \\
\hline$\varepsilon_{\mathrm{t}}\left(10^{-4}\right)$ & 1,88 & 1,87 & 1,85 & 0,13 \\
\hline$\varepsilon_{\mathrm{v}}\left(10^{-4}\right)$ & 2,92 & 2,03 & 2,01 & 0,68 \\
\hline $\mathrm{D}_{1}(0,01 \mathrm{~mm})$ & 242,50 & 185,10 & 164,60 & 0,68 \\
\hline
\end{tabular}

(*) Pavimento de referencia. 
La comparación de las respuestas estructurales, indicadas en la Tabla 4, presenta dos situaciones relevantes en la parte superior de la subrasante (en la profundidad de $0,68 \mathrm{~m}$ ):

- Reducción acentuada en la deflexión, cuando el $\mathrm{H}_{\text {REF }}$ pasó de $0,20 \mathrm{~m}$ a $0,40 \mathrm{~m}$, es decir, la deflexión de 242,50 (x 10-2 $\mathrm{mm}$ ) alcanzó 185,10 (x 10-2 mm). La guía SETRA establece 200 (x $10^{-2} \mathrm{~mm}$ ) para el control de ejecución cuando el módulo del soporte de la fundación corresponde a $50 \mathrm{MPa}$, pero el manual direcciona que para alcanzar ese valor es necesario la ejecución del refuerzo en la subrasante;

- El refuerzo de la subrasante igual a $0,60 \mathrm{~m}$ resulta en un $\varepsilon_{\mathrm{v}}$ igual a 2,01 (x $\left.10^{-4}\right)$, valor próximo al límite establecido en experimentos internacionales para pavimentos de larga duración $\left(\varepsilon_{\mathrm{v}}=2,00 \times 10^{-4}\right)$.

La condición de refuerzo estructural de la Figura 8 también puede ser verificada en el efecto de la deformación elástica, o sea, la deflexión en todo el pavimento, conforme la Figura 9.

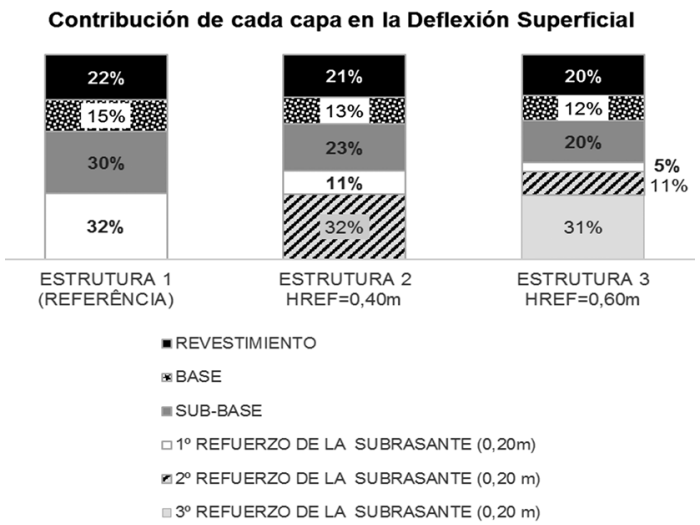

Figura 9. Contribución de las capas del suelo en la deflexión superficial $\left(\mathrm{D}_{0}\right)$.

En la Figura 9, se puede observar cómo las capas inciden en la deformación elástica (deflexión) del pavimento estudiado y cómo esa deflexión puede ser reducida con el aumento del $\mathrm{H}_{\mathrm{REF}}$. En la Estructura 1, se observa que las capas de base y sub-base contribuyen con un $45 \%$ en la deflexión en la superficie $\left(\mathrm{D}_{0}\right)$. Sin embargo, cuando el $\mathrm{H}_{\mathrm{REF}}$, pasa a tener $0,40 \mathrm{~m}$ (Estructura 2$)$ y $0,60 \mathrm{~m}$
(Estructura 3) ese porcentaje se reduce al 36\% y $32 \%$, respectivamente. Esta situación muestra que los pavimentos flexibles con subrasante débiles o frágiles poseen elevada deformación inicial y, si la deformación de esta capa (subrasante) es controlada, el pavimento tiende a ser más durable.

\section{CONCLUSIONES}

Los análisis de sensibilidad mostraron la relevancia de dos parámetros de rigidez, espesor y módulo elástico, tiene para cada capa en un pavimento flexible. Estos análisis demostraron que una pequeña variación en la capacidad estructural de las capas de revestimiento, base, sub-base y subrasante, puede ocasionar una considerable variación en los esfuerzos actuantes, afectando el desempeño del pavimento $\mathrm{y}$, consecuentemente, en su vida útil.

El módulo de elasticidad de la subrasante $\left(\mathrm{E}_{\mathrm{s}}\right)$ es el parámetro de rigidez más sensible en el comportamiento de un pavimento flexible. Esto significa que el cambio del $\mathrm{E}_{\mathrm{sl}}$ se refleja directamente en la deflexión en la superficie $\left(\mathrm{D}_{0}\right)$ y en la deformación de compresión en la parte superior de la subrasante $\left(\varepsilon_{\mathrm{v}}\right)$. Las guías y estudios internacionales como ALIANCE [4] y SETRA [5] establecen límites para estos esfuerzos y así obtener un pavimento de larga duración.

Para que estos pavimentos atiendan el ciclo de diseño completo, de modo que ocurra sólo intervenciones de carácter funcional, la subrasante debe ser protegida con un refuerzo, de espesor mínimo de $0,60 \mathrm{~m}$, material con módulo de resiliencia como mínimo a $100 \mathrm{MPa}$, que se denomina refuerzo de la subrasante. Esta solución redistribuye las deflexiones recuperables de las capas del pavimento y atiende una deflexión, medida en la parte superior de la subrasante, de 200 (x $10^{-2} \mathrm{~mm}$ ), valor exigido en el momento de la ejecución para subrasante de 50 $\mathrm{MPa}$, conforme la guía francesa [5]. La deformación permanente medida en la parte superior de la subrasante se aproxima a 2,00 (x 10-4), valor límite establecido en pavimento de larga duración [3-5].

Los módulos de elasticidad $\mathrm{E}_{\mathrm{rev}}$ y $\mathrm{E}_{\mathrm{b}}$ son sensibles a la deformación de tracción horizontal de la capa asfáltica $\left(\varepsilon_{t}\right)$, situación también observada por Mallick y El-Korchi [3]. Aunque en este estudio los esfuerzos $\varepsilon_{\mathrm{t}}$ presentaron poca sensibilidad en la reducción del espesor de las capas granulares, los estudios 
evidencian la importancia de considerar el estado de tensiones en capas granulares, principalmente cuando éstas son espesas. Las variaciones de los espesores de las capas de revestimiento $\left(\mathrm{H}_{\text {rev }}\right)$, base $\left(\mathrm{H}_{\mathrm{b}}\right)$ y sub-base $\left(\mathrm{H}_{\mathrm{sb}}\right)$ se mostraron sensibles al esfuerzo $\varepsilon_{\mathrm{v}}$, evidenciando una vez más la necesidad de proteger a la subrasante para el pavimento estudiado.

El refuerzo de la subrasante es una solución de refuerzo estructural, pues altera todo el estado de tensiones del pavimento flexible, amenizando principalmente las deformaciones verticales de la subrasante. Los resultados de este estudio mostraron que los pavimentos, con baja capacidad de soporte y dimensionados con espesores mínimos, en todas las capas, se consideran frágiles. De esta forma, los pavimentos con módulos de subrasante inferiores a $80 \mathrm{MPa}$, se debe prever como mínimo un refuerzo de $0,60 \mathrm{~m}$ de espesor. Sin embargo, el refuerzo de la subrasante para $\mathrm{E}_{\mathrm{sl}}$ menores de $30 \mathrm{MPa}$ no es viable, pues sería necesario un gran espesor de refuerzo. Para esta situación se debe seguir las sugerencias internacionales de tratamiento con aditivos para estabilización química que produzca mezclas estabilizadas con desempeño superior a capas con $\mathrm{E}_{\mathrm{sl}}$ $=50 \mathrm{MPa}$. Por lo tanto, concluyese que las sugerencias presentadas en este trabajo pueden contribuir a un proceso de dimensionamiento que haga la estructura de pavimento más resistente, durable y sustentable.

\section{REFERENCIAS}

[1] Confederação Nacional de Transporte. A importância do setor de transporte. Disponível em < http://pesquisarodoviascms.cnt.org.br// Relatorio\%20Geral/Pesquisa\%20CNT\%20 (2017)\%20-\%20ALTA.pdf >. Acesso em 07 de novembro de 2017.

[2] AASHTO. "Mechanistic-Empirical Pavement Design Guide", Interim Edition. A Manual of Practice. AASHTO. Washington, D. C. USA. 2008. p. 216. ISBN 978-1-56051-423-7.

[3] R.B. Mallick, T. El-Korchi. "Pavement Engineering Principles and Practice". $2^{\text {nd }}$ edition. CRC Press. New York, USA. 2013, p. 666. ISBN: 978-1-4398-7035-8.

[4] D. Newcomb. "Perpetual Asphalt Pavements - A Synthesis". Asphalt Pavement Alliance, 2010. URL: http://www.asphaltroads.org/ assets/_control/content/files/Perpetual_ Pavement_Synthesis.pdf
[5] SETRA/LCPC. "Conception et Dimensionnement des Structures de Chaussée Guide Technique". França. 1994.

[6] DNIT. "Manual de Pavimentação". Publicação IPR-719. Rio de Janeiro, Brasil. 2006.

[7] J. Medina e L.M.G. Motta. "Mecânica dos Pavimentos". Interciência Ltda. 3. ed. Rio de janeiro, Brasil, p. 368. 2015. ISBN: 978-85-7193-366-8.

[8] J.T. Balbo. "Pavimentação Asfáltica". Oficina de textos. 1. ed. São Paulo, Brasil, p. 558. 2007. ISBN: 978-85-86238-56-7.

[9] Y.H. Huang. "Pavement Analysis and Design". Pearson Prentice Hall. 2. ed. Upper Saddle River, New Jersey, USA. 2004. ISBN: 0-13-142473-4.

[10] W.J. Turnbull, C.R. Foster e R.G. Ahlvin. "Design of flexible pavements considering mixed loads and traffic volume". International Conference on the Structural Design of Asphalt Pavements Proceedings. University of Michigan, Ann Arbor, Estados Unidos. 1962.

[11] DNIT. "Manual de pavimentação. Departamento Nacional de Estradas e Rodagens". Rio de janeiro, Brasil. 1996.

[12] DNIT. "Manual de Restauração de Pavimentos Asfálticos". Publicação IPR-720. Rio de Janeiro, Brasil. 2006.

[13] DER/SP. "IP-DE-P00/001 - Projeto de Pavimentação". São Paulo, Brasil. 2006.

[14] S. Pinto. "Estudo do Comportamento à Fadiga de Misturas Betuminosas e Aplicação na Avaliação Estrutural de Pavimentos". COPPE/UFRJ, Rio de Janeiro, Brasil. 1991.

[15] E. Dorman e C. Metcalf. "Design Guide". Austroads Sidney. Sidney, Austrália. 1965.

[16] DNIT. "Classificação de solos tropicais para finalidades rodoviárias utilizando corposde prova compactados em equipamento miniatura". DNER-CLA 259. 06 p. Rio de Janeiro, Brasil. 1996.

[17] PMSP. "IP-05/2004 - Dimensionamento de Pavimentos Flexíveis Tráfego Meio Pesado, Pesado, Muito Pesado e Faixa Exclusiva de Ônibus”. 1994.

[18] G.S.R. Massenlli, C.E.L. Paiva, E. Cassio. "Análisis del Comportamiento de Pavimentos Flexibles con Subrasantes Débiles". $18^{\circ}$ Convención Científica de Ingeniería y Arquitectura. 2016. 